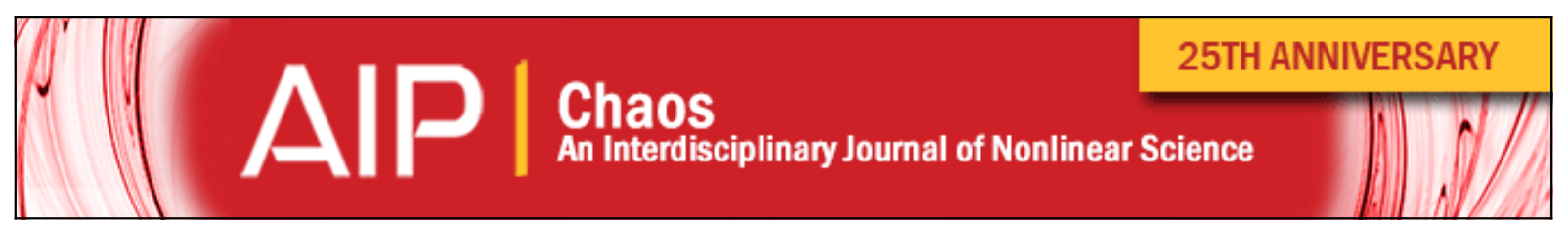

\title{
Expectation formation in an overlapping generation model with production
}

Fausto Cavalli and Ahmad Naimzada

Citation: Chaos 26, 033101 (2016); doi: 10.1063/1.4942798

View online: http://dx.doi.org/10.1063/1.4942798

View Table of Contents: http://scitation.aip.org/content/aip/journal/chaos/26/3?ver=pdfcov

Published by the AIP Publishing

\section{Articles you may be interested in}

Unbounded dynamics in dissipative flows: Rössler model

Chaos 24, 024407 (2014); 10.1063/1.4871712

Dynamics in a nonlinear Keynesian good market model

Chaos 24, 013142 (2014); 10.1063/1.4870015

Hierarchy of bifurcations in the truncated and forced nonlinear Schrödinger model

Chaos 15, 013107 (2005); 10.1063/1.1831591

Modeling of heat explosion with convection

Chaos 14, 263 (2004); 10.1063/1.1695211

Itinerant memory dynamics and global bifurcations in chaotic neural networks

Chaos 13, 1122 (2003); 10.1063/1.1601912

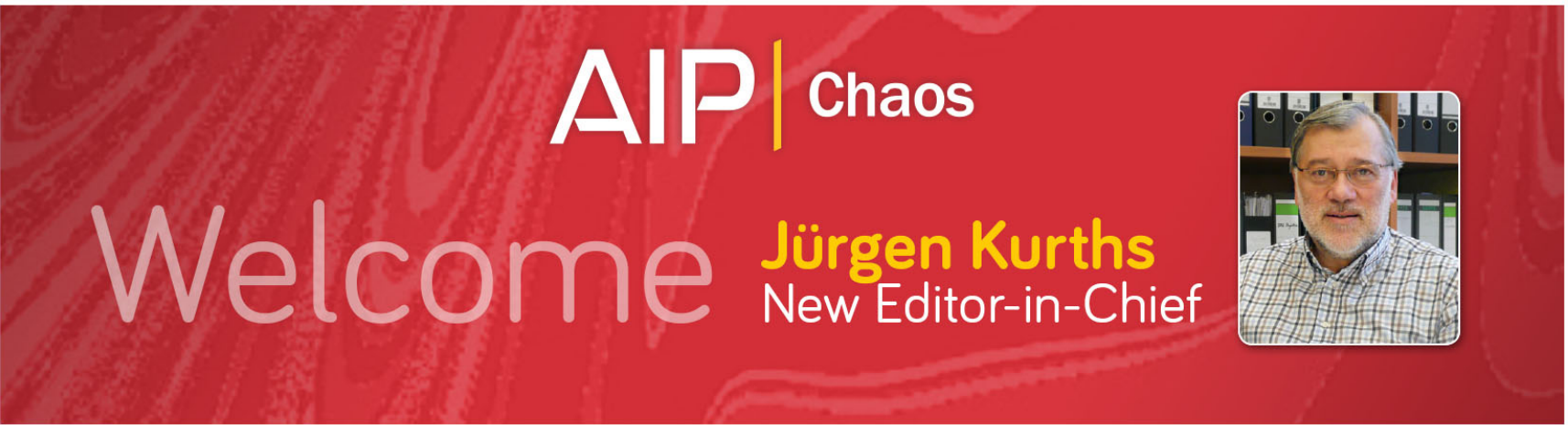




\title{
Expectation formation in an overlapping generation model with production
}

\author{
Fausto Cavallia) and Ahmad Naimzada ${ }^{\text {b) }}$ \\ Department of Economics, Management and Statistics, University of Milano-Bicocca, U6 Building, \\ Piazza dell' Ateneo Nuovo 1, 20126 Milano, Italy
}

(Received 7 October 2015; accepted 15 February 2016; published online 1 March 2016)

\begin{abstract}
In this paper, we investigate the dynamic properties of an overlapping generations' model with capital accumulation, in which agents work in both periods of life. We compare three different expectation mechanisms: perfect foresight, myopic foresight, and adaptive expectations, focusing, in particular, on this last one. We show that the steady state is the same under each mechanism, and we prove its global stability for perfectly foresighted agents. After investigating local stability conditions under myopic expectations, we study in detail the case of adaptive expectations. We show that, under both reduced rationality mechanisms, if the share of time devoted to labor in the second period of life is large enough, periodic and complex dynamics can occur. Moreover, deepening the investigation through numerical simulations, we study the global stability behavior under adaptive expectations. Such complex scenarios also include the coexistence between the stable steady state and a periodic or chaotic attractor, giving rise to multistability, which does not arise under myopic expectations. Finally, we provide some considerations about the possibility for the agents to improve their forecasts by observing the forecasting error time series. C) 2016 AIP Publishing LLC. [http://dx.doi.org/10.1063/1.4942798]
\end{abstract}

In overlapping generation (OLG) models, each agent lives a finite length of time which partially overlaps with that of other agents. The most usual and simple situation consists of agents which live for two periods, being "young" and "old", and only work when young. Since each period is usually assumed to correspond to $25 / 30$ years, in this framework, the agents actually work for 25/ 30 years. However, it is more realistic and topical to assume that working life can last for a longer time. To this end, we modify a classical OLG model so that a part of the second period of life is devoted to work. Moreover, a particular relevance on the resulting macroeconomic behaviors is represented by the expectation formation mechanism. Agents, in order to decide their optimal consumption and saving levels, have to "know" the future value of some economic variables, as interest rate and wages. The agents are often assumed to be completely rational, namely, to be able to exactly know such next period values. However, this assumption can be unrealistic. The agents more likely construct their expectations on the basis of current and/or past information. We show that the resulting model under reduced rationality expectation mechanisms can lead to non-convergent dynamics even considering a simple economic setting.

\section{INTRODUCTION}

Since the seminal work by Diamond (1965), OLG models have been extensively applied to the analysis of business cycles (Grandmont, 1985), economic growth and social security (de la Croix and Michel, 2002; Fanti and Gori, 2011; and Gori and Sodini, 2014), fertility related choice (Chen

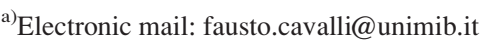

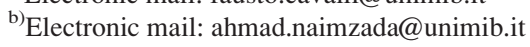

and Li, 2013 and Fanti and Gori, 2013), and fiscal and monetary policy (Blanchard and Fisher, 1989). A significant aspect in the overlapping generation structure is how agents form their future expectations about their second period of life, and how this affects the economic behavior in intertemporal macroeconomic models. For this reason, over the last two decades, interest in studying expectation formation mechanisms has grown. Indeed, perfect foresight usually provides the most stable evolutions. For instance, it is wellknown that if we assume logarithmic utility function, CobbDouglas production function, and perfectly foresighted agents, then the map describing the evolution of capital is increasing and concave, so that the resulting dynamic is globally convergent. However, the rationality requirement of perfect foresight can be unrealistic, as agents would more likely construct their expectations about the future on the basis of current and/or past information. For this reason, research focused on expectation formation mechanisms involving a reduced rationality degree, studying the differences in the resulting economic behaviors, in particular, with respect to rational expectations. Classical examples of reduced rationality expectations can be obtained if we assume that the agents expect that future prices will be the same as the current prices (myopic expectations) or if they try to adapt them on the basis of current and past information (adaptive expectations). We remark that research about expectation mechanisms is common to different economic contexts and can be found, for example, in the contributions by Hommes (1994, 1998)

The literature on stability of OLG models under different expectation mechanisms is quite wide; we mention only the contributions about pure exchange OLG models, as those by Bullard (1994), Duffy (1994), and Tuinstra (2003), and about OLG models with accumulation, as the works by de la 
Croix and Michel (2000), Chen et al. (2008), and Chen and Li (2008). In particular, de la Croix and Michel (2000) investigated whether the dynamics under simpler myopic expectations can suitably describe the evolution given by rational expectations, showing that, in general, this is false. They obtained general conditions under which myopic expectations cannot be used to characterize the dynamics under perfect foresight and provided a numerical example to show that both periodic and chaotic behaviors are possible. Later, Chen et al. (2008) enhanced the investigation focusing on constant elasticity of substitution (CES) utility function and Cobb-Douglas production function and compared the resulting models under perfect foresight, myopic, and adaptive expectations. After showing that under perfect foresight we have a stable, globally converging behavior, they provided rigorous proofs about the occurrence of chaotic dynamics when myopic and adaptive expectations are used. They proved that if the inter-temporal elasticity of substitution is sufficiently high, the resulting dynamics under myopic expectations exhibit topological chaos. They also studied adaptive expectations with expectation weight near 1 (namely, when they can be seen as a small perturbation of myopic dynamics), showing that if both the inter-temporal elasticity of substitution and total factor productivity are sufficiently large, then the resulting dynamics exhibit entropic chaos. We notice that, because of the CES utility function, the analytic expression of the steady state equilibrium is not explicitly available, and consequently, it is not possible to obtain explicit local stability thresholds in terms of the economic parameters. Moreover, since the model under adaptive expectations is only studied as a perturbation of the myopic case, it is not possible to say, whether and when adaptive expectations can provide convergent dynamics. We notice that if a logarithmic utility function is considered, the resulting dynamics under myopic and adaptive expectations are always convergent.

In our contribution, we study an OLG model inspired by that reported in the book by de la Croix and Michel (2002), in which the agents can partially work in the second period of life too. We consider logarithmic utility function and Cobb-Douglas production function, with agents that supply one unit of labor in the first period and $h<1$ unit in the second one, and we study and compare the dynamics under perfect foresight, myopic, and adaptive expectations, paying specific attention to this last case. Unlike in de la Croix and Michel (2000) and Chen et al. (2008), thanks to the simpler form of the utility function, we can provide the explicit expression of both the steady state (which does not depend on the expectation mechanisms) and the stability thresholds above which the dynamics do not converge. We notice that in Gori and Sodini (2011), an OLG model in which the agents partially work in both periods of life is considered. The amount of labor they supply is endogenously determined. Besides some differences in the economic context, our contribution differs from that by Gori and Sodini (2011) for the following aspects. The main difference lies in the exogenous (in the present work) and endogenous (in Gori and Sodini (2011)) way in which the amount of labor supplied for the second period of life is determined. Moreover, in the present paper, the agents always supply one unit of labor when young, while in Gori and Sodini (2011), they supply only a fraction of time. In Gori and Sodini (2011), the agents are assumed to have perfect foresight, and the goal is to study and show the occurrence of indeterminacy, nonlinear dynamics, and multiplicity of equilibria due to the effect of public provision of investments in health. Conversely, we mainly aim to study, in a simple economic context, the consequences of the possibility for the agents to work for a longer time, consisting not only of the whole first period (when agents are young) but also of an initial fraction of the second one (before agents retire). In particular, we want to investigate the effects induced on the dynamics by reduced rationality expectations, focusing on adaptive expectations.

We show that under perfect foresight the dynamics are globally convergent, while unstable dynamics can arise under both myopic and adaptive expectations. We obtain the stability threshold in terms of $h$, of the discount factor of the preference function and of the capital share to output in the Cobb-Douglas production function, in addition to the expectation weight in the case of adaptive expectations. We show that increasing $h$ may have a destabilizing effect on the steady state equilibrium, for both myopic and adaptive expectations. Conversely, increasing the discount factor and the capital share to output allows steady state to be stable for larger values of $h$. We remark that the resulting map under perfect foresight and myopic expectations is structurally similar to that obtained in Fanti and Gori (2013), in which a different economic context with endogenous fertility and public pensions is studied.

Most significant results concern the study of adaptive expectations. Indeed, if dynamics are unstable under myopic foresight, adaptive expectations with suitably small expectation weight allow for recovering steady state stability. However, we show that nonconvergent behaviors are possible even if the expectation weight is very small, and not necessarily only when it is close to 1 . In particular, this occurs when the capital share to output is suitably small. Furthermore, we show that when agents adopt adaptive expectations, the local stability alone does not allow characterizing the convergence, since the stable steady state equilibrium can coexist with a complex (periodic or chaotic) attractor, which is induced by the expectation mechanisms. Such situation, which is also known in literature as "corridor stability" (Leijonhufvud, 1973; Dohtani et al., 2007; and Bischi and Naimzada, 2014), shows that a stable steady state and dynamics in which small perturbations are strengthened by the nonlinearity of the system can actually coexist. Moreover, it shows that a more refined mechanism (adaptive expectation), even if allows for recovering local stability of the steady state, may not guarantee the convergence toward the steady state equilibrium. In this sense, the local stability analysis alone is not sufficient to catch the whole behavior and has to be supported by global investigations. Finally, considering the configurations for which complex dynamics arise, we want to understand whether the agents could be able to improve their forecasts. To this end, following the approach used in Hommes (2013), we investigate the autocorrelation function (ACF) of the forecast error time series 
obtained under adaptive expectations, finding that, in general, it is difficult to improve the forecasts.

The paper is organized as follows. In Section II, we introduce the OLG model. In Section III, we study dynamics under the assumption of perfect and myopic foresight. In Section IV, we investigate adaptive expectations, focusing on stability and convergence of dynamics. In Section V, we investigate the possibility to improve forecasts under adaptive expectations. In Section VI, we present some conclusions and future perspectives.

\section{MODEL}

We consider a finite-horizon, discrete time overlapping generations model with productive capital. Agents, who live for two periods, supply one unit of labor in the first period and $0<h<1$ unit in the second one. As noticed in the book by de la Croix and Michel (2002), $h<1$ can be interpreted as part time work or early retirement. We assume this latter interpretation. We suppose that the agents have identical Cobb-Douglas preferences, and that they only care about their consumptions in each period of life. Moreover, we assume that there is no population growth, and we normalize the population size to 1 .

We denote the consumptions in the first and second periods of life of agents born at time $t$, respectively, by $c_{t}$ and $c_{t+1}$, so that preferences are given by the logarithmic utility function

$$
u=\log c_{t}+\beta \log c_{t+1},
$$

where $\beta \in(0,1)$ is the discount factor.

Let us introduce the expected real interest rate $R_{t+1}^{e}$, the first period life wage $\omega_{t}$, the expected second period life wage $\omega_{t+1}^{e}$, and the saving decision by the young agent $s_{t}$. The budget constraints are then

$$
\omega_{t} \geq c_{t}+s_{t}
$$

for young agents and

$$
c_{t+1} \leq s_{t} R_{t+1}^{e}+h \omega_{t+1}^{e},
$$

for old agents, so that each young generation maximizes its utility (1) under constraints (2) and (3), provided that both $c_{t} \geq 0$ and $c_{t+1} \geq 0$. This implies that if agents work in the second period too, the income used for consumption when old comes not only from savings made when young but also from wage when old. Taking into account constraints (2) and (3), we can rewrite the utility function (1) as

$$
u=\log \left(\omega_{t}-s_{t}\right)+\beta \log \left(s_{t} R_{t+1}^{e}+h \omega_{t+1}^{e}\right),
$$

so that, given $R_{t+1}^{e}$ and $\omega_{t+1}^{e}$, the optimal saving decision by the young agent results

$$
s_{t}=\frac{\beta}{1+\beta} \omega_{t}-\frac{h}{1+\beta} \frac{\omega_{t+1}^{e}}{R_{t+1}^{e}} .
$$

We notice that, from (4), the optimal decision of savings for the young generation is increasing in the first period life wage and decreasing in the expected second period life wage.

As in Chen et al. (2008), we assume Cobb-Douglas production function $Y_{t}=A K_{t}^{\alpha} L_{t}^{1-\alpha}$, with $A>0$ and $\alpha \in(0,1)$, where $K_{t}$ and $L_{t}$ are, respectively, capital and labor used for production at period $t$, while parameters $A$ and $\alpha$, respectively, represent the total factor productivity and the capital share to output. Consequently, the production function in intensive form is $y_{t}=A k_{t}^{\alpha}$, where $y_{t}$ and $k_{t}$, respectively, represent per capita output and per capita capital, and factor prices are

$$
\omega_{t}=A(1-\alpha) k_{t}^{\alpha}
$$

and

$$
R_{t}=A \alpha k_{t}^{\alpha-1}
$$

where $R_{t}$ represents the real interest rate. The capital market equilibrium condition implies that $k_{t+1}=s_{t}$, so that, given an initial condition $k_{0}$, the equilibrium trajectory $\left\{k_{t}\right\}_{t \geq 0}$ is given by

$$
k_{t+1}=\frac{\beta}{1+\beta} \omega_{t}-\frac{h}{1+\beta} \frac{\omega_{t+1}^{e}}{R_{t+1}^{e}} .
$$

We notice that Equation (7) is structurally similar to the equilibrium trajectory obtained in the work by Fanti and Gori (2013) (see p. 1216). In this work, an OLG model with endogenous fertility and public pensions is considered. Both technology and preferences are the same as those considered in the present paper. The economic settings in Fanti and Gori (2013) and in our contribution are indeed different. However, at least from the mathematical point of view, budget constraints (2) and (3) bear resemblance to those imposed in Fanti and Gori (2013), which, respectively, are

$$
c_{1, t}+s_{t}+q \omega_{t} n_{t}=\omega_{t}(1-\theta)
$$

for a young individual of generation $t$ and

$$
c_{2, t+1}=R_{t+1}^{e} s_{t}+\theta \omega_{t+1}^{e}\left[(1-w) \bar{n}_{t}+w n_{t}\right],
$$

which represents the constraint at time $t+1$ of an individual of generation $t$. We notice that $c_{1, t}$ (respectively, $c_{2, t+1}$ ) represents the consumption when young (respectively, old), $q$ is the percentage of working income devoted to child rearing, $w$ is the weight of individual fertility $n_{t}$ with respect to average fertility $\bar{n}_{t}$, and $\theta$ is the constant contribution rate. In particular, we notice that (9) formally reduces to (3) by setting $h=\theta\left[(1-w) \bar{n}_{t}+w n_{t}\right]$. The economic meaning of (3) and (9) is indeed different. In both models, the second period consumption $c_{t+1}$ depends on $\omega_{t+1}^{e}$. However, in (3), $\omega_{t+1}^{e}$ represents the second period expected life wage of old agents of generation $t$, who supply $h \in(0,1)$ unit of labor. Conversely, in (9), $\omega_{t+1}^{e}$ is the first period expected life wage of young agents of generation $t+1$. Moreover, $\theta[(1-w)$ $\left.\bar{n}_{t}+w n_{t}\right]$ is an endogenous terms, connected to fertility, which represents the tax receipt. Both $h \omega_{t+1}^{e}$ and $\theta \omega_{t+1}^{e}\left[(1-w) \bar{n}_{t}+w n_{t}\right]$ similarly act on the second period 
expected income, so that the resulting optimization problem is similar and, under the same preferences, provides structurally similar equilibrium trajectories. We remark that since production function is also the same, the resulting wage rate at time $t+1$ is the same for both old agents of generation $t$ and young agents of generation $t+1$, and this means that adopting the same expectation mechanism in both the present model and in the model studied in Fanti and Gori (2013) leads to similar dynamical equations, which, however, describe different economic situations and have different interpretations.

To make dynamics explicit for per capita capital $k_{t}$, we need to specify expected values $\omega_{t+1}^{e}$ and $R_{t+1}^{e}$. As in Chen et al. (2008), we investigate three different expectation mechanisms: perfect foresight, myopic, and, especially, adaptive expectations.

If agents are assumed to be perfectly foresighted, we have $\omega_{t+1}^{e}=\omega_{t+1}$ and $R_{t+1}^{e}=R_{t+1}$, namely,

$$
\omega_{t+1}^{e}=A(1-\alpha) k_{t+1}^{\alpha}, \quad R_{t+1}^{e}=A \alpha k_{t+1}^{\alpha-1},
$$

and the dynamic of $k_{t}$ is implicitly represented by

$$
k_{t+1}=\frac{\beta}{1+\beta} A(1-\alpha) k_{t}^{\alpha}-\frac{h}{1+\beta} \frac{A(1-\alpha) k_{t+1}^{\alpha}}{A \alpha k_{t+1}^{\alpha-1}},
$$

which can be easily rewritten in an explicit form as

$$
k_{t+1}=g\left(k_{t}\right)=\frac{\alpha \beta(1-\alpha) A}{\alpha(1+\beta)+h(1-\alpha)} k_{t}^{\alpha} .
$$

Conversely, as in de la Croix and Michel (2000), we can assume myopic expectations, namely, agents expect that both the second period real interest rate and life wage will remain the same as in the current period $\left(\omega_{t+1}^{e}\right.$ $=\omega_{t}, R_{t+1}^{e}=R_{t}$ ), which gives

$$
\omega_{t+1}^{e}=A(1-\alpha) k_{t}^{\alpha}, \quad R_{t+1}^{e}=A \alpha k_{t}^{\alpha-1} .
$$

Putting (12) in (7), we obtain the one-dimensional equation

$$
k_{t+1}=m\left(k_{t}\right)=\frac{\beta}{1+\beta} A(1-\alpha) k_{t}^{\alpha}-\frac{h}{1+\beta} \frac{(1-\alpha)}{\alpha} k_{t} .
$$

We notice that both Equations (11) and (13) have a similar structure to dynamical equations in Fanti and Gori (2013) under the corresponding perfect foresight and myopic expectations. Differently from de la Croix and Michel (2000) and Fanti and Gori (2013), we also consider adaptive expectations, in which the expected values are formed on the basis of current and past information with decreasing weights. In what follows, for simplicity, we assume that the same weight $\lambda \in(0,1]$ is used in forming expectations for both $\omega_{t+1}^{e}$ and $R_{t+1}^{e}$, so that

$$
\begin{aligned}
\omega_{t+1}^{e} & =\sum_{i=0}^{\infty} \lambda(1-\lambda)^{i} \omega_{t-i}=(1-\lambda) \omega_{t}^{e}+\lambda \omega_{t} \\
& =(1-\lambda) \omega_{t}^{e}+\lambda A(1-\alpha) k_{t}^{\alpha}=f_{2}\left(\omega_{t}^{e}, k_{t}\right),
\end{aligned}
$$

and

$$
\begin{aligned}
R_{t+1}^{e} & =\sum_{i=0}^{\infty} \lambda(1-\lambda)^{i} R_{t-i}=(1-\lambda) R_{t}^{e}+\lambda R_{t} \\
& =(1-\lambda) R_{t}^{e}+\lambda \alpha A k_{t}^{\alpha-1}=f_{3}\left(R_{t}^{e}, k_{t}\right) .
\end{aligned}
$$

We notice that if $\lambda=1$, we recover myopic expectations (12). If we put (14) and (15) into (7), we obtain the evolution equation for per capita capital

$$
\begin{aligned}
k_{t+1} & =\frac{\beta}{1+\beta} A(1-\alpha) k_{t}^{\alpha}-\frac{h}{1+\beta} \frac{(1-\lambda) \omega_{t}^{e}+\lambda A(1-\alpha) k_{t}^{\alpha}}{(1-\lambda) R_{t}^{e}+\lambda \alpha A k_{t}^{\alpha-1}} \\
& =f_{1}\left(\omega_{t}^{e}, R_{t}^{e}, k_{t}\right) .
\end{aligned}
$$

The resulting model under adaptive expectations is then represented by the three-dimensional system $T\left(\omega_{t}^{e}, R_{t}^{e}, k_{t}\right)$ $=\left(f_{1}\left(\omega_{t}^{e}, R_{t}^{e}, k_{t}\right), f_{2}\left(\omega_{t}^{e}, k_{t}\right), f_{3}\left(R_{t}^{e}, k_{t}\right)\right)$

$T:\left\{\begin{array}{l}k_{t+1}=\frac{\beta}{1+\beta} A(1-\alpha) k_{t}^{\alpha}-\frac{h}{1+\beta} \frac{(1-\lambda) \omega_{t}^{e}+\lambda A(1-\alpha) k_{t}^{\alpha}}{(1-\lambda) R_{t}^{e}+\lambda \alpha A k_{t}^{\alpha-1}}, \\ \omega_{t+1}^{e}=(1-\lambda) \omega_{t}^{e}+\lambda A(1-\alpha) k_{t}^{\alpha} \\ R_{t+1}^{e}=(1-\lambda) R_{t}^{e}+\lambda \alpha A k_{t}^{\alpha-1} .\end{array}\right.$

Since system (17) actually encompasses the model under myopic foresight, as the dynamics of $k_{t+1}$ provided by (17) for $\lambda=1$ are the same of those provided by (13), in what follows the greatest relevance will be given to the study of adaptive expectations. However, to first deal with a simple situation, we investigate local stability of model under myopic expectations too. We remark that both (13) and (17) could provide negative values of $k_{t+1}$ for some initial data, which is indeed unfeasible. In what follows, we assume that initial data are chosen so that $k_{t}$ stays positive for any $t>0$. Some analytical details about the positivity of trajectories of (13) are reported in Proposition 3, while some numerical considerations are provided for system (17).

We notice that if we considered an economy characterized by constant intertemporal elasticity of substitution (CIES) preferences with inter-temporal elasticity of substitution $\sigma$ and CES technology with degree of substitutability of the inputs $\rho$ (see de la Croix and Michel (2002) and Fanti and Gori (2013)), the resulting equilibrium trajectory would be

$$
k_{t+1}=\frac{\beta^{\sigma}}{\beta^{\sigma}+\left(R_{t+1}^{e}\right)^{1-\sigma}} \omega_{t}-\frac{h}{\beta^{\sigma}+\left(R_{t+1}^{e}\right)^{1-\sigma}} \cdot \frac{\omega_{t+1}^{e}}{\left(R_{t+1}^{e}\right)^{\sigma}},
$$

with factor prices given by $R_{t}=a A\left[a+(1-a) k_{t}^{\rho}\right]^{-(1+\rho) / \rho}$ and $\omega_{t}=A(1-a)\left[a k_{t}^{-\rho}+(1-a)\right]^{-(1+\rho) / \rho}$. In this case, the resulting model under perfect foresight is only implicitly defined, while, due to the lack of an explicit analytic expression for the steady state equilibrium, the stability under myopic and adaptive expectations cannot be analytically investigated. A deep numerical investigation of this case is out of the scope of the present work, which aims to deal with the Cobb-Douglas/logarithmic economy.

First, we specify the possible nontrivial steady state of models (11), (13), and (17) in the following proposition, whose proof is straightforward. 
Proposition 1. At the steady state, both perfect foresight, myopic, and adaptive expectations have the same strictly positive capital steady state

$$
k^{*}=\left(\frac{\alpha \beta(1-\alpha) A}{\alpha(1+\beta)+h(1-\alpha)}\right)^{\frac{1}{1-\alpha}},
$$

to which expected wage $\omega^{e, *}=A(1-\alpha)\left(k^{*}\right)^{\alpha}$ and interest rate $R^{e, *}=A \alpha\left(k^{*}\right)^{\alpha-1}=(\alpha(1+\beta)+h(1-\alpha)) /(\beta(1-\alpha))$ correspond.

The previous result shows that the nontrivial steady state for per capita capital is the same under each expectation mechanism. We notice that $k^{*}$ is strictly positive for any $\alpha, \beta \in(0,1)$ and $h \in(0,1)$. Finally, we remark that, from Proposition 1, we have an inverse relation between the steady state value of per capita capital $k^{*}$ and the quantity of labor in the second period. This result comes from the negative relation between saving and second period wage, as the possible income in the second period of life induces agents to save a reduced amount of capital in the first period.

\section{DYNAMICS UNDER PERFECT AND MYOPIC FORESIGHT}

In this section, we study models (11) and (13). In particular, we notice that the study of the local stability under myopic expectations is a particular case of that under adaptive expectations. Its investigation is then preparatory to the analysis of the more general case of adaptive expectations, performed in Section IV.

First, we consider the case of perfect foresight. Function $g\left(k_{t}\right)$ defined in (11) is indeed positive, strictly increasing (as $\alpha>0$ ) and concave (as $\alpha<1$ ). The resulting trajectories are then well-defined and consist of feasible values of $k$ for any initial datum and parameter configurations. The stability of $k^{*}$ is precised in the following proposition.

Proposition 2. Unique nontrivial steady state $k^{*}$ is globally asymptotically stable for (11).

Proof. Recalling that a steady state is locally asymptotically stable if and only if $\left|g^{\prime}\left(k^{*}\right)\right|<1$, since $g^{\prime}(k)=$ $\left(\alpha^{2} \beta(1-\alpha) A k^{\alpha-1}\right) /(\alpha(1+\beta)+h(1-\alpha))$, we have that $g^{\prime}\left(k^{*}\right)=\alpha \in(0,1)$, which implies local stability. The global stability is a straightforward consequence of the monotonicity and concavity of $g$.

The previous result confirms those reported in de la Croix and Michel (2000) and Chen et al. (2008) about rational expectations, in the case of CES utility function and with $h=0$.

Now we study the case of myopic expectations. We start noticing that function $m(k)$ is the sum of a nonlinear increasing power function of degree $\alpha<1$ and a linear decreasing term, and so it is a one hump map. From an economic perspective, this is due to the fact that current savings are increasing with respect to current income, but decreasing with respect to future income, which is discounted with the expected interest rate, and both depend on the current capital through myopic expectations. This is essentially due to the presence of $h>0$. We remark that, conversely, the map obtained when $h=0$, which is identical to that in Chen et al. (2008) when preferences are logarithmic, is always increasing. The following proposition investigates the stability of the positive steady state (18).

Proposition 3. The unique, nontrivial steady state $k^{*}$ is locally asymptotically stable for any $h \in(0,1)$ if

$$
3 \alpha+\alpha \beta+\alpha^{2} \beta-1 \geq 0 .
$$

When condition (19) is not satisfied, then $k^{*}$ is locally asymptotically stable provided that

$$
h<h_{1}=\frac{\alpha(1+\alpha)(1+\beta)}{(1-\alpha)^{2}} .
$$

If the previous condition is violated, the steady state undergoes a cascade of period doublings for $h \in\left[h_{1}, h_{2}\right)$, where

$$
h_{2}=\frac{1+\beta}{(1-\alpha)^{2}} \alpha^{\frac{1-2 \alpha}{1-\alpha}} .
$$

When $h \geq h_{2}$, we may have $k_{t+1} \leq 0$.

Proof. Steady state $k^{*}$ is stable if and only if $\left|m^{\prime}\left(k^{*}\right)\right|<1$. Since

$$
m^{\prime}(k)=\frac{\beta}{1+\beta} A(1-\alpha) \alpha k^{\alpha-1}-\frac{h(1-\alpha)}{\alpha(1+\beta)},
$$

we have $m^{\prime}\left(k^{*}\right)=\alpha-\left(h(1-\alpha)^{2}\right) /(\alpha(1+\beta))$. Condition $\left|m^{\prime}\left(k^{*}\right)\right|<1$ leads to the couple of inequalities $h>$ $\alpha(\alpha-1)(1+\beta) /(1-\alpha)^{2}$, which is indeed true since $\alpha<1$, and $h<h_{1}$. Since $h<1$, steady state $k^{*}$ is then unconditionally stable when $h_{1} \geq 1$, which provides (19). However, we need to investigate whether, under conditions (19) and (20), we have $k_{t}>0$ for any $t$. Map $m(k)$ is unimodal and concave, so to have $m(k)>0$, we need $0<k<\hat{k}$, where

$$
\hat{k}=\left(\frac{\alpha \beta A}{h}\right)^{\frac{1}{1-\alpha}}
$$

realizes $m(\hat{k})=0$. Due to the unimodal shape of $m(k)$, to have $k_{t}>0$ for any $t>0$, we need that $m\left(k_{M}\right)<\hat{k}$, where $k_{M}$ is the per capita capital for which function $m$ attains its maximum value. From (21), we have

$$
k_{M}=\left(\frac{A \alpha^{2} \beta}{h}\right)^{\frac{1}{1-\alpha}},
$$

and

$$
m\left(k_{M}\right)=\frac{(1-\alpha)^{2}}{1+\beta} \frac{h}{\alpha^{2}}\left(\frac{A \alpha^{2} \beta}{h}\right)^{\frac{1}{1-\alpha}} .
$$

Imposing positivity condition $m\left(k_{M}\right)<\hat{k}$, after some easy algebraic manipulations, we find

$$
\frac{(1-\alpha)^{2}}{1+\beta} h \alpha^{\frac{-1+2 \alpha}{1-\alpha}}<1
$$

or, equivalently, $h<h_{2}$, which represents the condition under which trajectories stay positive for any initial $k_{0}$.

Now we study the compatibility of conditions (19) and (20) with $h<h_{2}$. We notice that (19) can be rewritten as 


$$
\beta \geq \frac{1-3 \alpha}{\alpha^{2}+\alpha},
$$

which is possible only if the r.h.s. is smaller than 1, namely, for $\alpha>\sqrt{5}-2$. From $h_{2}$ and (22), we have

$$
h_{2}>r(\alpha)=\frac{\alpha^{-\frac{\alpha}{1-\alpha}}}{\alpha+1}>1 \text {, }
$$

since $r(\alpha)$ and $\lim _{\alpha \rightarrow \sqrt{5}-2} r(\alpha) \approx 1.26$. This means that when we have unconditional stability with respect to $h$, trajectories do not cross unfeasible regions.

Conversely, if (19) is not satisfied, we have that $h_{1}<h_{2}$. In fact, it can be equivalently rewritten as $\alpha(1+\alpha)<$ $\alpha^{(1-2 \alpha) /(1-\alpha)}$, which it is easy to see that it is true for $\alpha \in(0,1)$. This means that when conditional stability (20) is satisfied, $k_{t}$ stays positive. Conversely, when (20) is violated, we need $h<h_{2}$.

The previous proposition states that stability is guaranteed when the quantity of time devoted to labor in the second period is suitably small, depending on parameters $\alpha$ and $\beta$. Condition (19) means that if the capital share to output is sufficiently large $(\alpha \geq 1 / 3)$, then $k^{*}$ is stable for any $\beta$ independently of $h$. Moreover, if $0<(1-3 \alpha) /\left(\alpha^{2}+\alpha\right)<1$ (i.e., if $\sqrt{5}-2<\alpha<1 / 3), k^{*}$ can be again unconditionally stable with respect to $h$, but only for a suitably large value of discount factor $\beta$. Finally, if $\alpha \leq \sqrt{5}-2$, then the steady state cannot be unconditionally stable. In this case, increasing $h$, we can have that trajectories could cross unfeasible region $k<0$. As already remarked, Equation (13) is structurally similar to that in Fanti and Gori (2013) under myopic expectations, both being regulated by one hump maps. The resulting local stability behavior is then similar. For example, in Fanti and Gori (2013), parameter $\theta$ representing the constant contribution rate has a destabilizing role, which resembles that of $h$ in the present model. The economic interpretation of the result is indeed different. In our contribution, as already noticed, current savings increase with respect to current income and decrease with respect to future incomes. If the current saving component is more relevant with respect to the future income component, the fixed point is locally asymptotically stable. Conversely, as expected future incomes become increasingly significant, map (13) becomes decreasing and increasingly steep in $k=k^{*}$ and can produce non-monotonic and non-convergent dynamics. The effect is mainly determined by the presence of $h$, which weights the decreasing term in (13), and instabilities occur when the capital share to output is relatively small with respect to the discount factor, as summarized by condition (20).

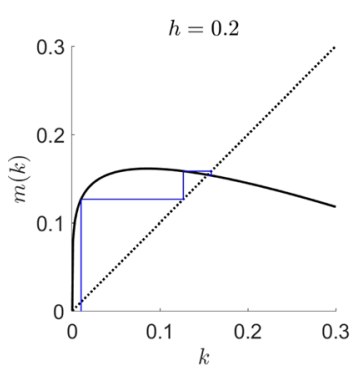

(a)

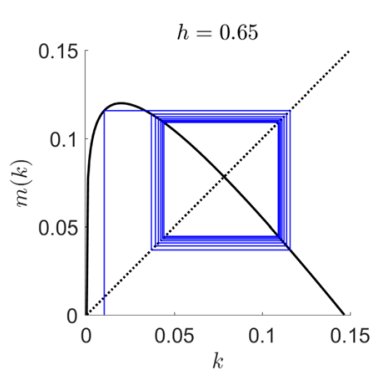

(b)

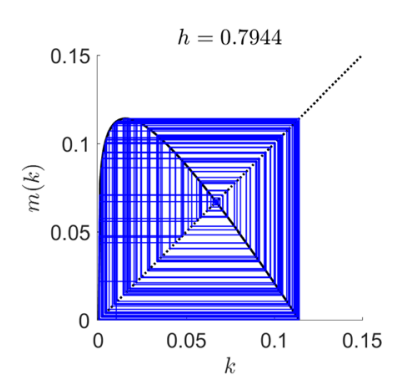

(c)

We remark that in Fanti and Gori (2013) no evidences about multistability phenomena are mentioned, as well as our numerical investigations confirm for the present model under myopic expectations (see also Figure 2).

In Figure 1, we report the plot of map (13) and the corresponding dynamics when $A=1, \alpha=0.2$, and $\beta=0.7$, for which, recalling Proposition 3 , we are in the situation of conditional stability. As $h$ increases ( $h=0.2,0.65$, and 0.7944$)$, we, respectively, have convergent, periodic, and chaotic dynamics. As we can see from Figure 1(c), if we increase $h$ above 0.7944 , we would have $k_{t}<0$ for some $t$. In Figure 2(a), we represent, using different colors, the values of $\min \left\{h_{1}, 1\right\}$ with respect to $\alpha$ and $\beta$. The yellow regions, in which we have $\min \left\{h_{1}, 1\right\}=1$, correspond to those $(\alpha, \beta)$ for which we have unconditional stability with respect to $h$, while to the right of the solid line we have stability only in increasingly smaller intervals of $h$, with blue representing $h_{1}=0$.

In Figure 2(b), we report a bifurcation diagram obtained setting $A=1, \alpha=0.1$, and $\beta=0.2$, on varying $h$. We notice that for these parameters, we have that $k^{*}$ is stable for $h \in$ $\left(0, h_{1}=0.4125\right)$, outside which a cascade of period doublings occurs, leading to chaos. For $h>h_{2}=0.5140, k_{t}$ becomes negative.

\section{DYNAMICS UNDER ADAPTIVE EXPECTATIONS}

In this section, we investigate the steady state equilibrium stability under adaptive expectations. Since it is the main focus of the present paper, after studying the local asymptotic stability of the steady state in Proposition 4, we deeply investigate through simulations qualitative aspects of global stability, focusing on the coexistence of multiple attractors and on their basins of attraction.

Proposition 4. Under adaptive expectations, the steady state $\left(k^{*}, \omega^{e, *}, R^{e, *}\right)$ of $(17)$ is locally asymptotically stable for any $h \in(0,1)$ and for any $\lambda \in(0,1]$ provided that $\alpha, \beta$ satisfy condition (19). Conversely, when $\alpha$ and $\beta$ are such that $3 \alpha+\alpha \beta+\alpha^{2} \beta-1<0$, the steady state is locally asymptotically stable for any $h \in(0,1)$ if $\lambda \in(0, \hat{\lambda}(\alpha)]$ with

$$
\hat{\lambda}(\alpha)=\frac{2 \alpha}{\alpha+1} .
$$

If $\lambda \in(\hat{\lambda}(\alpha), 1]$, then $\left(k^{*}, \omega^{e, *}, R^{e, *}\right)$ is locally asymptotically stable provided that

$$
h<\frac{\alpha(\alpha+1)(\beta+1)(2-\lambda)}{(1-\alpha)(\lambda-2 \alpha+\alpha \lambda)} .
$$

FIG. 1. Maps $m(k)$ and resulting dynamics for different values of $h$ and for $A=1, \alpha=0.2$, and $\beta=0.7$. (a) Convergent dynamics, (b) periodic dynamics, and (c) chaotic dynamics. 
Proof. To prove steady state stability, we need the Jacobian matrix $J$ of system (17), whose elements are

$$
\begin{gathered}
\partial_{k} f_{1}=\frac{A \alpha \beta k^{\alpha-1}(1-\alpha)}{\beta+1}-\frac{A \alpha h k^{\alpha-1} \lambda(1-\alpha)}{(\beta+1)\left(R(1-\lambda)+A \alpha k^{\alpha-1} \lambda\right)} \\
-\frac{A \alpha h k^{\alpha-2} \lambda(1-\alpha)\left(w(1-\lambda)+A k^{\alpha} \lambda(1-\alpha)\right)}{(\beta+1)\left(R(1-\lambda)+A \alpha k^{\alpha-1} \lambda\right)^{2}}, \\
\partial_{\omega} f_{1}=-\frac{h(1-\lambda)}{(\beta+1)\left(R(1-\lambda)+A \alpha k^{\alpha-1} \lambda\right)}, \quad \partial_{R} f_{1}=\frac{h(1-\lambda)\left(w(1-\lambda)+A k^{\alpha} \lambda(1-\alpha)\right)}{(\beta+1)\left(R(1-\lambda)+A \alpha k^{\alpha-1} \lambda\right)^{2}}, \\
\partial_{k} f_{2}=A \alpha k^{\alpha-1} \lambda(1-\alpha), \quad \partial_{\omega} f_{2}=1-\lambda, \quad \partial_{R} f_{2}=0, \\
\partial_{k} f_{3}=-A \alpha k^{\alpha-2} \lambda(1-\alpha), \quad \partial_{\omega} f_{3}=0, \quad \partial_{R} f_{3}=1-\lambda .
\end{gathered}
$$

Evaluating $J$ at $\left(k^{*}, \omega^{e, *}, R^{e, *}\right)$, we find

$$
J^{*}=\left(\begin{array}{ccc}
-\frac{\left(B h \lambda-A \alpha^{2} \beta\right)(1-\alpha)}{B \alpha(\beta+1)} & -\frac{B h(1-\lambda)}{A \alpha(\beta+1)} & \frac{B^{\frac{2-\alpha}{1-\alpha}} h(1-\alpha)(1-\lambda)}{A \alpha^{2}(\beta+1)} \\
\frac{A \alpha \lambda(1-\alpha)}{B} & 1-\lambda & 0 \\
-\frac{A \alpha \lambda(1-\alpha)}{B^{\frac{2-\alpha}{1-\alpha}}} & 0 & 1-\lambda
\end{array}\right),
$$

in which we set

$$
B=\frac{A \alpha \beta(1-\alpha)}{h+\alpha(\beta-h+1)} .
$$

We make use of stability conditions

$$
\left\{\begin{array}{l}
1-\left|\operatorname{det}\left(J^{*}\right)\right|>0 \\
1-\mathrm{M}\left(J^{*}\right)+\operatorname{tr}\left(J^{*}\right) \operatorname{det}\left(J^{*}\right)-\operatorname{det}^{2}\left(J^{*}\right)>0 \\
1+\mathrm{M}\left(J^{*}\right)-\operatorname{tr}\left(J^{*}\right)-\operatorname{det}\left(J^{*}\right)>0 \\
1+\mathrm{M}\left(J^{*}\right)+\operatorname{tr}\left(J^{*}\right)+\operatorname{det}\left(J^{*}\right)>0
\end{array}\right.
$$

reported in Brooks (2004), in which $\mathrm{M}$ is the sum of the three principal minors $M_{1}, M_{2}$, and $M_{3}$ of the Jacobian matrix $J^{*}$. Since

$$
\begin{gathered}
M_{1}\left(J^{*}\right)=(1-\lambda)^{2}, \quad M_{2}\left(J^{*}\right)=\frac{(1-\lambda)(\alpha+h+\alpha \beta-\alpha h-h \lambda+\alpha h \lambda)}{\beta+1}, \\
M_{3}\left(J^{*}\right)=\frac{(1-\lambda)\left(\alpha h-h \lambda+\alpha^{2} \beta-\alpha^{2} h+\alpha^{2}+2 \alpha h \lambda-\alpha^{2} h \lambda\right)}{\alpha(\beta+1)},
\end{gathered}
$$

we have

$$
\mathrm{M}\left(J^{*}\right)=\frac{(1-\lambda)\left(\alpha+\alpha \beta+2 \alpha h-\alpha \lambda-h \lambda+2 \alpha^{2} \beta-2 \alpha^{2} h+2 \alpha^{2}-\alpha \beta \lambda+\alpha h \lambda\right)}{\alpha(\beta+1)} .
$$

Moreover, we have

$$
\operatorname{tr}\left(J^{*}\right)=\frac{2 \alpha+2 \alpha \beta+\alpha h-2 \alpha \lambda-h \lambda+\alpha^{2} \beta-\alpha^{2} h+\alpha^{2}-2 \alpha \beta \lambda+\alpha h \lambda}{\alpha(\beta+1)},
$$




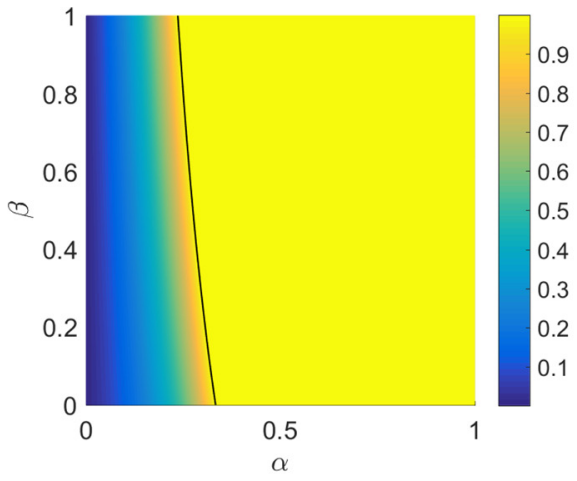

(a)

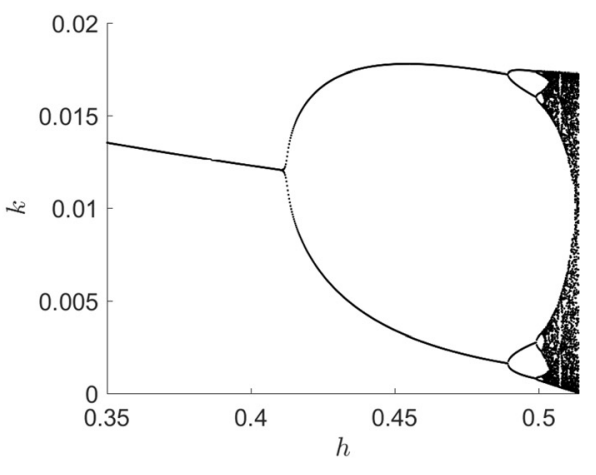

(b)

and

$$
\operatorname{det}\left(J^{*}\right)=\frac{(h+\alpha(\beta-h+1))(1-\lambda)^{2}}{\beta+1} .
$$

We notice that since $\operatorname{det}\left(J^{*}\right)>0$, the first condition in (24) reduces to $1-\operatorname{det}\left(J^{*}\right)>0$, which can be rewritten as

$$
\frac{-h(1-\alpha)(1-\lambda)^{2}+(\beta+1)\left(1-\alpha(1-\lambda)^{2}\right)}{\beta+1}>0 .
$$

The denominator is indeed positive, and we have

$$
\begin{aligned}
& -h(1-\alpha)(1-\lambda)^{2}+(\beta+1)\left(1-\alpha(1-\lambda)^{2}\right) \\
& >-(1-\alpha)(1-\lambda)^{2}+\left(1-\alpha(1-\lambda)^{2}\right) \\
& \quad=\lambda(2-\lambda)>0,
\end{aligned}
$$

so $1-\operatorname{det}\left(J^{*}\right)>0$ is always satisfied. After some algebraic manipulations, the second condition of (24) becomes

$$
\frac{c_{1} h^{2}+c_{2} h+c_{3}}{\alpha(\beta+1)^{2}}>0
$$

where

$$
\begin{aligned}
& c_{1}=-\lambda(1-\alpha)^{2}(1-\lambda)^{2}(\alpha \lambda-2 \alpha+1), \\
& c_{2}=\lambda(1-\alpha)(\beta+1)(1-\lambda)(2 \alpha \lambda-4 \alpha+1)(\alpha \lambda-\alpha+1), \\
& c_{3}=\alpha \lambda(\beta+1)^{2}(2-\lambda)(\alpha \lambda-\alpha+1)^{2} .
\end{aligned}
$$

Let us introduce the values for which the r.h.s. of (25) vanishes

$$
\begin{aligned}
& h_{A}=\frac{c_{2}+\sqrt{c_{2}^{2}-4 c_{1} c_{3}}}{2 c_{1}}=\frac{(\beta+1)(\alpha \lambda-\alpha+1)}{(1-\alpha)(1-\lambda)}, \\
& h_{B}=\frac{c_{2}-\sqrt{c_{2}^{2}-4 c_{1} c_{3}}}{2 c_{1}}=\frac{\alpha(\beta+1)(2-\lambda)(\alpha \lambda-\alpha+1)}{(1-\alpha)(1-\lambda)(-\alpha \lambda+2 \alpha-1)} .
\end{aligned}
$$

Since the denominator of (25) is indeed positive, condition (25) is satisfied when $h_{B}<h<h_{A}$ if $(\alpha \lambda-2 \alpha+1)>0$ and when $h<h_{A} \vee h>h_{B}$ if $(\alpha \lambda-2 \alpha+1)<0$. We notice that when $(\alpha \lambda-2 \alpha+1)>0$, we have $h_{B}<0$. Moreover, we always have $h_{A}>1$ since

FIG. 2. (a) Stability with myopic expectations. The colors represent the maximum value of $h$ below which $k^{*}$ is stable. The solid line represents condition (19). To its right (yellow region), we have unconditional stability. (b) Bifurcation diagram on varying $h$. which means that condition (25) is always satisfied for all $h \in(0,1)$.

The third condition of (24) is equivalent to

$$
\frac{\lambda^{2}(1-\alpha)(\alpha(1-h)+h+\alpha \beta)}{\alpha(\beta+1)}>0
$$

and it is indeed true.

The last condition of (24) is equivalent to

$$
\begin{aligned}
& \frac{(2-\lambda)}{\alpha(\beta+1)}[h(1-\alpha)(-\lambda+2 \alpha-\alpha \lambda) \\
& \quad+\alpha(\alpha+1)(\beta+1)(2-\lambda)]>0,
\end{aligned}
$$

which is satisfied provided that the term within square brackets is positive. This means that if $(-\lambda+2 \alpha-\alpha \lambda)>0$, or equivalently (23), we have unconditional stability with respect to $h$. If the previous relation is false, then we need

$$
h<\frac{\alpha(\alpha+1)(\beta+1)(2-\lambda)}{(1-\alpha)(\lambda-2 \alpha+\alpha \lambda)} .
$$

The previous relation is true for any $h \in(0,1)$ if its r.h.s. is larger or equal than 1 or, equivalently, when

$$
\frac{-\left(\alpha+\alpha \beta+\alpha^{2} \beta+1\right) \lambda+\left(4 \alpha+2 \alpha \beta+2 \alpha^{2} \beta\right)}{(1-\alpha)(\lambda-2 \alpha+\alpha \lambda)} \geq 0,
$$

which is solved by

$$
\lambda \leq \frac{2 \alpha(2+\beta+\alpha \beta)}{(\alpha+1)(1+\alpha \beta)} .
$$

The previous relation is true for any $\lambda \in(0,1]$ provided that its r.h.s. is greater or equal to 1 , namely, when

$$
\frac{3 \alpha+\alpha \beta+\alpha^{2} \beta-1}{\alpha+\alpha \beta+\alpha^{2} \beta+1} \geq 0,
$$

which requires $3 \alpha+\alpha \beta+\alpha^{2} \beta-1 \geq 0$. Noticing that, since $2+\beta+\alpha \beta>1+\alpha \beta$, the r.h.s. of (26) is indeed larger than $\hat{\lambda}(\alpha)$, we can conclude. 


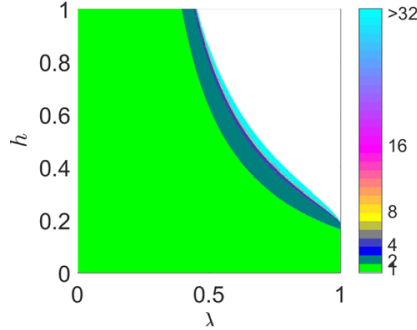

(a)

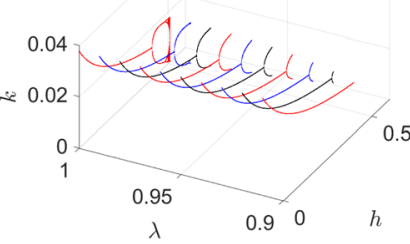

(b)

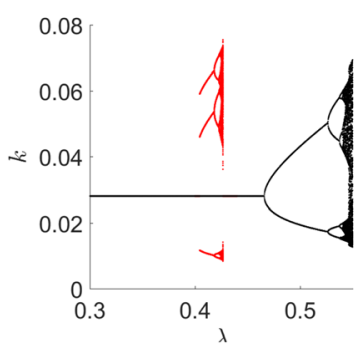

(c)

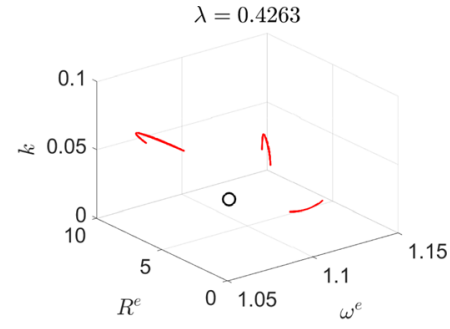

(d)

FIG. 3. (a) Bifurcation diagram for (17) with respect to $h, \lambda$. The white region represents parameter settings for which trajectories cross unfeasible regions. The green color is used for the stable region, while the remaining colors are used for attractors consisting of more than a single point. (b) Snapshots of the bifurcation diagram with respect to $h$ for different values of $\lambda$. (c) Bifurcation diagrams for two different initial data, showing multistability, with the steady state coexisting with a periodic or chaotic attractor. (d) Space phase plot of the steady state (circle) and of the chaotic attractor.

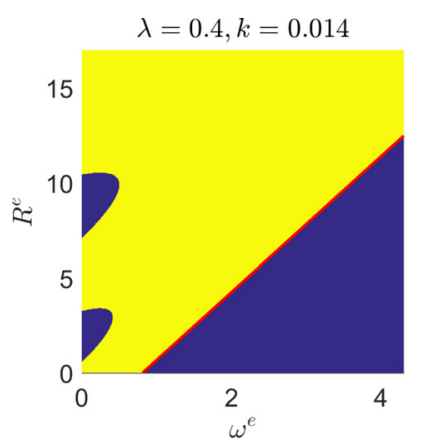

(a)

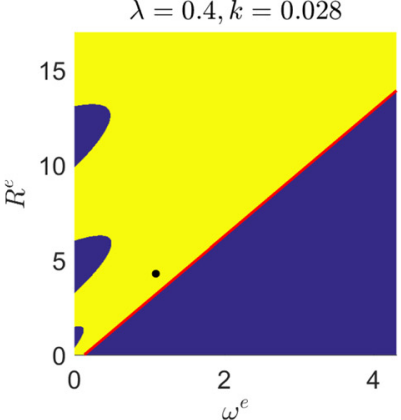

(b)

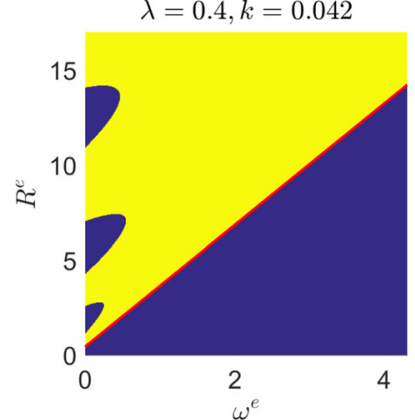

(c)

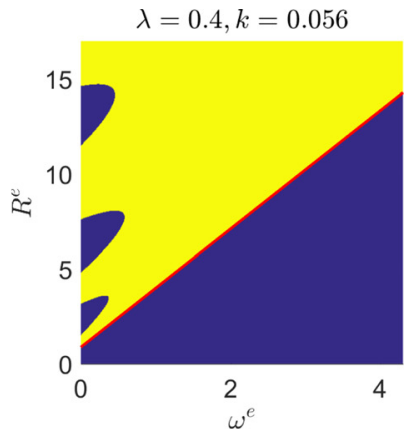

(d)

FIG. 4. Sections along planes $k=$ const of the basins of attraction related to map (17). The blue color is used for initial data which provide trajectories in which $k$ become negative. The yellow color represents initial data which provide trajectories converging toward the steady state equilibrium (represented by the black dot).

The previous proposition explains the role of $h$ and $\lambda$ for the steady state equilibrium stability. First, we notice that for any $\alpha, \beta$, we can always find a suitably small $\lambda$ for which the steady state is unconditionally stable for any $h \in(0,1)$. However, it is not possible to find a value of $\lambda$ which guarantees stability for any economic setting, as, from (23), if $\alpha$ becomes increasingly small, $\hat{\lambda}$ vanishes too. To investigate the dynamics when the steady state loses its stability, in Figure 3(a), we report a two parameters bifurcation diagram, obtained for the same configuration ( $A=1, \alpha=0.1$, and $\beta=0.2$ ) used for the simulation under myopic foresight of Figure 2. As we can see, when the steady state becomes unstable (outside the green region), it incurs a cascade of period doubling bifurcations, if $\lambda$ or $h$ is increased, which leads to chaos (inside the light blue region). In Figure 3(b), we report snapshots for ten different values of $\lambda \in[0.91,1]$ of the bifurcation diagram with respect to $h$, in which it is evident the stabilizing role of $\lambda$. We remark that as $\lambda$ decreases, weights $\lambda(1-\lambda)^{i}$ in (14) and (15) give more and more uniform relevance to all the past prices (for a similar behavior, we refer to Bischi and Naimzada (1997)).

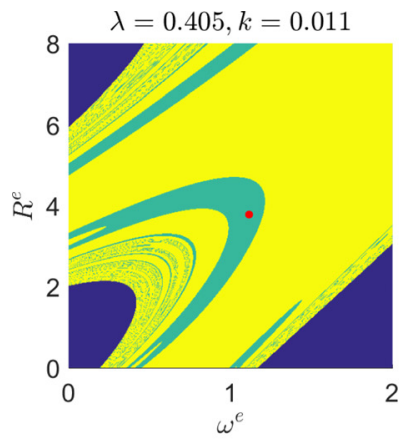

(a)

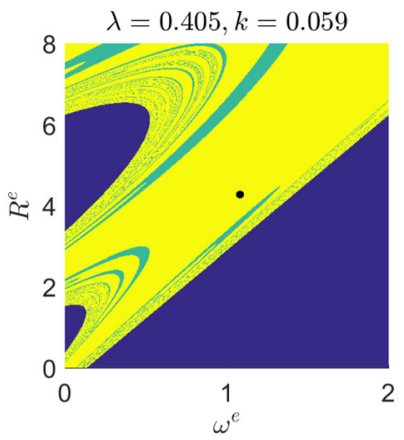

(b)

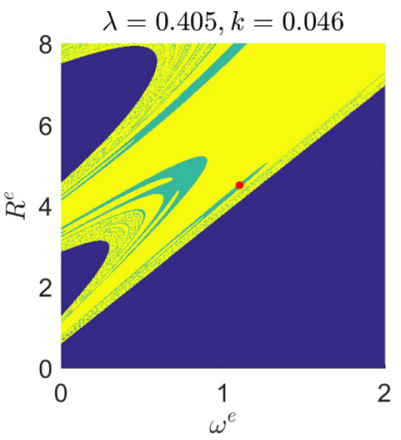

(c)

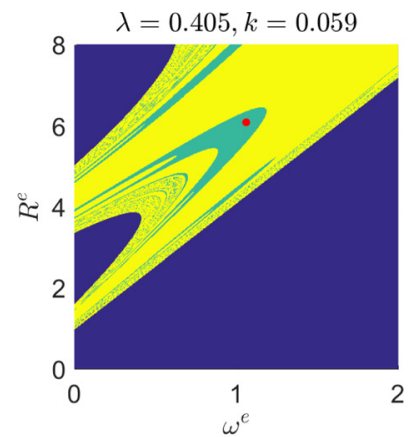

(d)

FIG. 5. Sections along planes $k=$ const of the basins of attraction related to map (17). The blue color is used for initial data which provide trajectories in which $k$ become negative. The yellow color represents initial data which provide trajectories converging toward the steady state (represented by the black dot). The light blue color represents initial data which provide trajectories converging toward the period-3 attractor (red dots). 


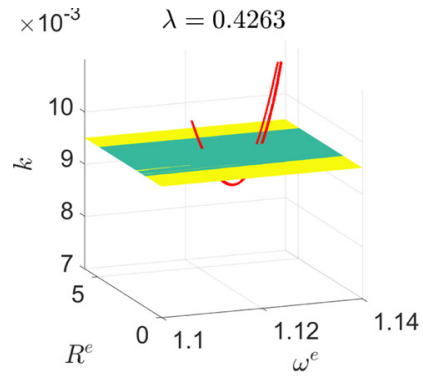

(a)

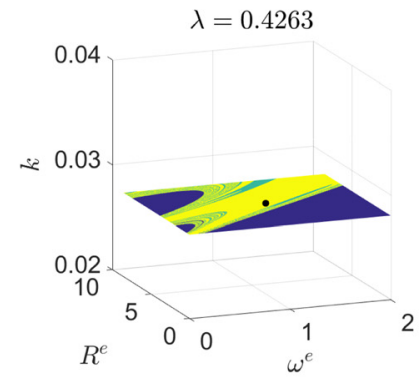

(b)

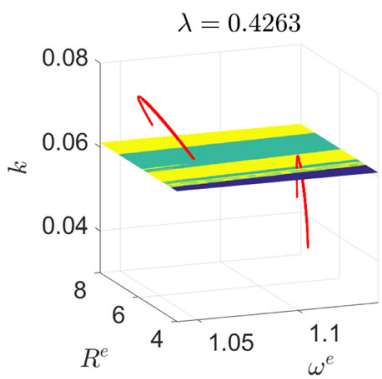

(c)

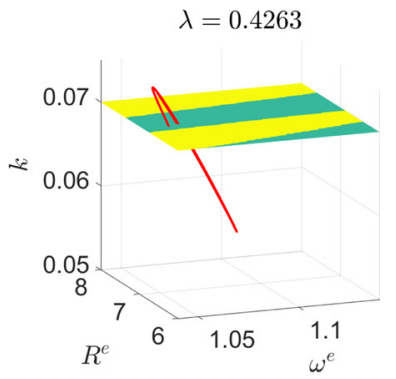

(d)

FIG. 6. Sections along planes $k=$ const of the basins of attraction related to system (17). The blue color is used for initial data which provide trajectories in which $k$ becomes negative. The yellow color represents initial data which provide trajectories converging toward the steady state (represented by the black dot). The light blue color represents initial data which provide trajectories converging toward the three pieces chaotic attractor (red color).

Even if Proposition 4 states that decreasing $\lambda$ improves local stability, convergence toward steady state equilibrium may be not guaranteed for any initial condition which provides positive trajectories. In Figures 3(c) and 3(d), we give evidence of the occurrence of multistability when adaptive expectations are used. If we consider $A=1.71, \alpha=0.1$, $\beta=0.2$, and $h=0.72$, for which the steady state is $\left(k^{*}, \omega^{e, *}, R^{e, *}\right)=(0.028,1.077,4.267)$ and we choose initial values $k_{0}=0.03, w_{0}^{e}=0.9$, and $R_{0}^{e}=3.9$, trajectories converge to the steady state for $\lambda<0.4656$. However, for a small perturbation of the initial expected prices (for example, if we take $\left.w_{0}^{e}=1.2, R_{0}^{e}=4.1\right)$, trajectories converge toward a different attractor for $\lambda \in[0.405,0.4265]$. This means that improving the learning mechanism (in this case replacing simple myopic foresight with slightly more refined adaptive expectations) can indeed allow stabilizing the steady state, but can also introduce complex multistability phenomena which lead trajectories far from the steady state, toward an attractor which, as we checked, is not present with myopic foresight and is induced by the expectation mechanism itself. To understand this, we perform a simulative global analysis of the basins of attraction. Since we are dealing with a three dimensional system, the basins of attraction are three dimensional regions of the phase space. To represent them, in what follows, we will plot sections cut along planes $k=$ const. Referring to the scenario of Figure 3(c), in Figure 4, we report the basins of attraction for $\lambda=0.4$ and for different values of $k$. In this case, for any initial value $\left(k_{0}, \omega_{0}, R_{0}\right)$, we have only two possibilities: $k_{t}$ becomes negative for some $t$ and the trajectory becomes unfeasible (blue color), or it converges to the steady state equilibrium (yellow color). In particular, we notice that the initial conditions belonging to the triangular blue region below the red line are those for which the first iterate has $k<0$. To show this, we notice that $T(k, \omega, R)=\left(k_{0}, \omega_{0}, R_{0}\right)$ has at most one real solution, and that the preimage of $\left(0, \omega_{0}, R_{0}\right)$ with $k_{0}<0, \omega_{0}>0$, and $R_{0}>0$ is obtained by solving $f_{1}(k, \omega, R)<0$ and satisfies

$$
\omega>\frac{A \beta k^{\alpha}(1-\alpha)}{h} R-A k^{\alpha}(1-\alpha) \frac{\lambda}{1-\lambda}\left(1-\frac{A \alpha \beta k^{\alpha-1}}{h}\right) .
$$

The remaining limited blue regions correspond to the successive preimages of the region described by (27). If we slightly increase $\lambda$, as reported in the bifurcation diagram of Figure $3(\mathrm{c})$, we have that the steady state equilibrium coexists with a period-3 attractor. In Figure 5, we report the sections along planes $k=0.011,0.046$, and 0.059 , to which each point of the attractor, respectively, belongs, and plane $k=0.028$ on which the steady state equilibrium lies. As we can see, we have that the basin of attraction of the coexisting period-3 attractor (light blue) is immersed in the basin of attraction of the steady state (yellow color) and consists of several unconnected regions, some of them with complicated topology and scrambled boundaries. This means that very close initial data (or a small perturbation of an initial datum) can provide very different dynamical evolutions. A similar situation can be seen in Figure 6, in which we report some blow-ups of sections of the basins of attraction when they are crossed by the three-piece, three dimensional coexisting chaotic attractors. We notice that we can have initial data which are "close" to the steady state (for example, when $\lambda=0.405$ and $\left(k, \omega^{e}, R^{e}\right)=(0.059,1.08,3.6)$ and provide trajectories that do not converge toward the steady state and initial data

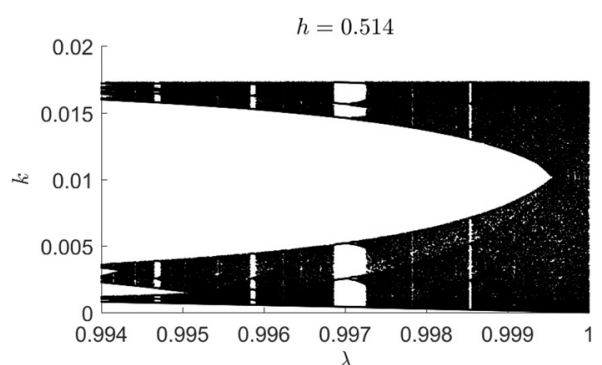

(a)

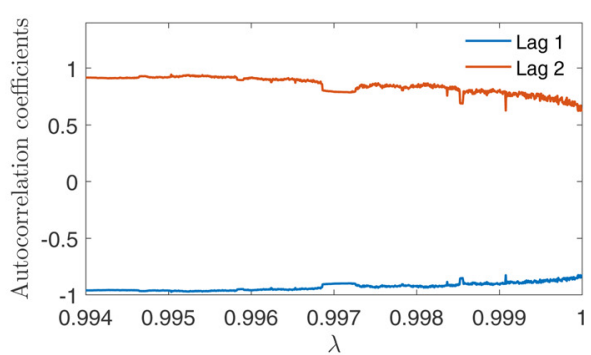

(b)
FIG. 7. (a) Bifurcation plot of $k$ for (17) for $\lambda>0.994$. (b) Plot of first (blue) and second (red) autocorrelation coefficient for $e_{\omega, t}$. 


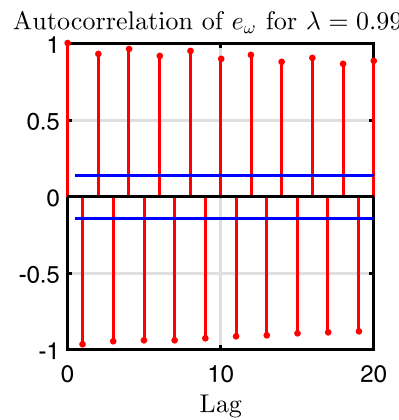

(a)

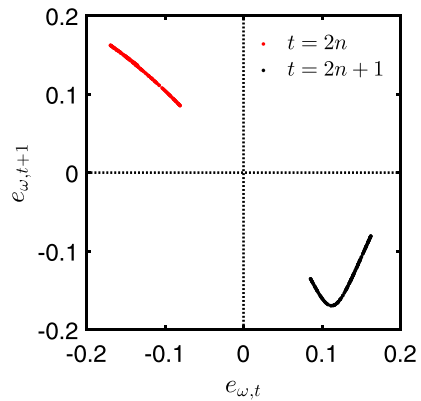

(b)

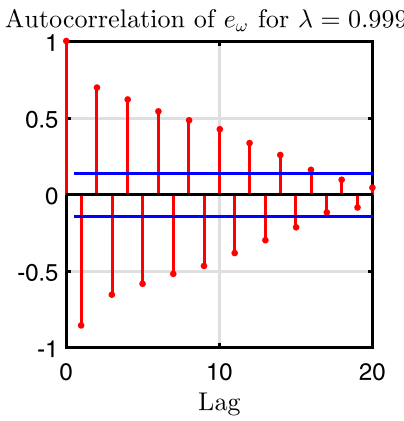

(c)

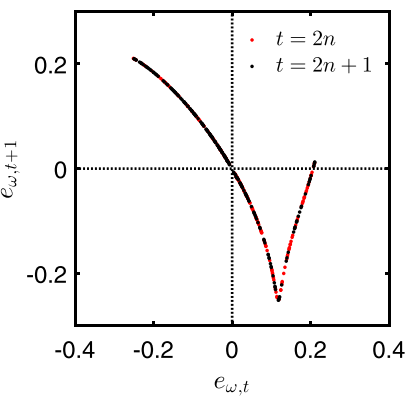

(d)

FIG. 8. (a) and (b) Autocorrelation plot of $e_{\omega, t}$ and plot of $e_{\omega, t+1}$ versus $e_{\omega, t}$ for $\lambda=0.9952<0.9996$. (c) and (d) Autocorrelation plot of $e_{\omega, t}$ and plot of $e_{\omega, t+1}$ versus $e_{\omega, t}$ for $\lambda=0.99976>0.9996$

which are "far" from the steady state (for example, when $\lambda=0.405$ and $\left.\left(k, \omega^{e}, R^{e}\right)=(0.059,2,8)\right)$ and provide trajectories that converge toward the steady state. The previous considerations show that different kinds of asymptotic behaviors are possible under adaptive expectations. When the steady state equilibrium is stable, convergence is possible only for initial values sufficiently close to the steady state and that a global investigation of the properties of the map is essential to have a full picture of the situation. Multistability has been investigated in depth in economic literature, and it has sometimes been referred to as "corridor stability" (see, for example, Leijonhufvud (1973), Dohtani et al. (2007), and Bischi and Naimzada (2014)).

\section{CHAOTIC DYNAMICS AND AUTOCORRELATION OF FORECASTING ERRORS}

When the agents do not have perfect foresight, they can make forecasting errors $e_{\omega, t}=\omega_{t}-\omega_{t}^{e}$ and $e_{R, t}=R_{t}-R_{t}^{e}$ at each time step. When dynamics are convergent, expectation errors vanish as the trajectory approaches the steady state, so the expectation mechanism becomes increasingly reliable. However, as we noticed in Sections III and IV, both myopic and adaptive expectations are not able to guarantee convergence for any parameter configurations, and periodic and chaotic trajectories can occur. In this section, we want to cast a glance at the possibility for the agents to improve their forecasts looking at the behavior of the past forecasting errors. Can they, observing past errors, forecast future expectation errors, on the basis of information coming from $e_{\omega, t}$ and $e_{R, t}$, in order to improve their expectation mechanism? If state variables follow a cyclical pattern, it is reasonable that, after a suitable number of periods, the agents become aware of the periodic nature of the trajectories and can be able to suitably correct their expectations.

When trajectories become chaotic, the scenario is indeed more problematic. In this case, we focus on adaptive expectations. As shown in Hommes (2013), a way for the agents to improve their forecasts can be the analysis of the time series of errors by means of the ACF. We remark that in what follows we only focus on $e_{\omega, t}$. We checked that the behavior of $e_{R, t}$ is qualitatively the same, so all the consideration are valid for $e_{R, t}$ too.

In Figure 7(a), we report a blow-up of the bifurcation plot for $h=0.514$ reported in Figure 3(b), for $\lambda>0.994$, namely, when chaotic dynamics arise. In Figure 7(b), we plot the same values of autocorrelation coefficients at lags 1 and 2 for the time series of $e_{\omega, t}$, for the values of $\lambda$ used in the bifurcation diagram reported in Figure 7(a). As we can see, the first autocorrelation coefficient is always negative, while the second one is always positive. For $\lambda<0.9996$, this pattern simply reveals that the chaotic attractor is made of two pieces, and that the state variables alternatively move from a piece to the other. In Figure 8(a), we report the autocorrelation plot obtained for $\lambda=0.9952$, whose oscillating pattern is common to those obtained for any $\lambda<0.9996$. In Figure $8(\mathrm{~b})$, we plot $e_{\omega, t+1}$ versus $e_{\omega, t}$, using red (respectively, black) color when $t$ is even (respectively, odd). The plot clearly shows that $e_{\omega, t}$ alternatively assumes positive and negative values and, since all points lie in either second or fourth quadrant, that a positive error is always followed by a negative one. This suggests that agents can be able to forecast the sign of the next period error, the value of which however changes. When $\lambda>0.9996$, the two pieces of the attractor merged, but there is still a significant persistence of the errors in regions with opposite signs, which however overlap. In Figure 8(c), we report the autocorrelation plot obtained for $\lambda=0.99976$, which is decreasingly oscillating. If we look at Figure 8(d), where we report the plot of $e_{\omega, t+1}$ versus $e_{\omega, t}$, we can see that now it is no more true that a positive error is always followed by a negative one, as we can find both red and black dots in the second and fourth quadrants. Moreover, some points lie in the first quadrant, showing the existence of consecutive positive errors. In this case,

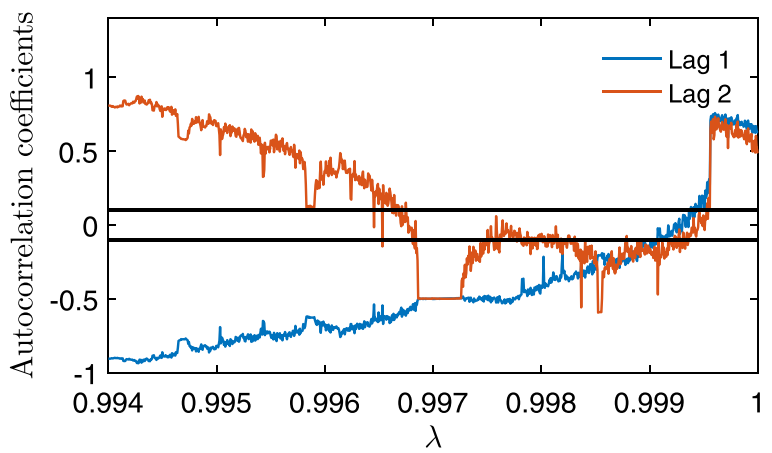

FIG. 9. Plot of first (blue) and second (red) order autocorrelation coefficients for $e_{\omega, t}^{0,2}$. 


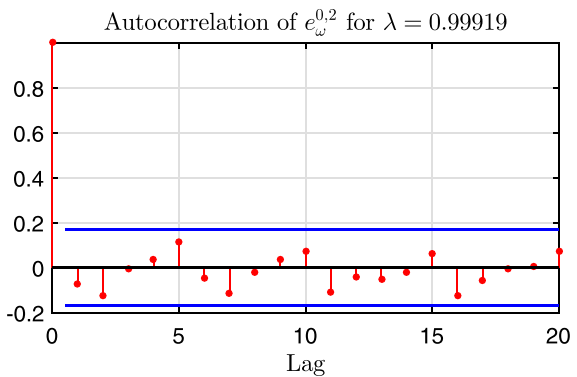

(a)

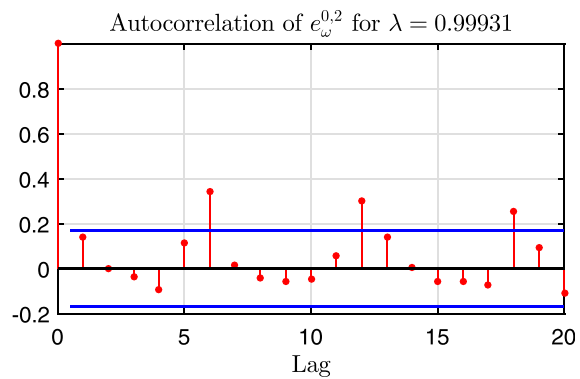

(b)
FIG. 10. (a) Autocorrelation coefficients of $e_{\omega, t}^{0,2}$ for $\lambda \approx 0.99919$ are negligible and are similar to a stochastic process. (b) Significant autocorrelation coefficients of $e_{\omega, t}^{0,2}$ for $\lambda \approx 0.999226$ occur at lags 6,12 , and 18 . it seems hard that agents be able to improve their forecasts observing the behavior of the time series of errors.

Conversely, if we consider $\lambda<0.9996$, we may wonder if observing the dynamics restricted to each piece of the attractor can provide some further information. This means that an agent, after having noticed that the forecasting error he will make at time $t+1$ has the same sign of that at time $t-1$ (and not of that at time $t$ ), can look at the time series restricted to $t-1, t-3, \ldots$ to try to forecast the error at time $t+1$. He can actually investigate about the existence of a correlation among even (respectively, odd) times only. To this end, we can subdivide the errors into two subsets

$$
e_{\omega, n}^{0,2}=\omega_{2 n}-\omega_{2 n}^{e}, \quad e_{\omega, n}^{1,2}=\omega_{1+2 n}-\omega_{1+2 n}^{e},
$$

where $e_{\omega, n}^{0,2}$ (respectively, $e_{\omega, n}^{1,2}$ ) consists of the forecasting errors made when $t=2 n$ is even (respectively, $t=2 n+1$ is odd). In Figure 9, we report the values of the autocorrelation coefficients at lags 1 and 2 for the time series of $e_{\omega, t}^{0,2}$ obtained for the same values of $\lambda$ used for the bifurcation plot reported in Figure 7. We verified that the plot computed for $e_{\omega, t}^{1,2}$ is very similar. As we can see, the scenario is more articulated with respect to Figure 7(b). In particular, among the different combinations, we notice that coefficients can

- have opposite signs (for example, when $\lambda=0.995$ );

- be both negative $(\lambda=0.997)$;

- have small absolute values $(\lambda=0.9994)$;

- be both positive $(\lambda=0.9997)$.

This shows a quite wide range of possibilities. The richness of dynamics, which are very sensitive to the expectation weight, cannot be, in general, easily "understood" in order to improve forecasts. In Figure 10, we report two different situations. If we look at the ACF of $e_{\omega, t}^{0,2}$ for $\lambda=0.99919$ (Figure 10(a)), we indeed have that the time series is indistinguishable from a random process, as all autocorrelations coefficients are very small. In this case, the dynamic is qualitatively the same obtained superimposing a stochastic noise to a period- 2 cycle. However, if we consider $\lambda=0.999226$ (Figure 10(b)), we notice significant autocorrelations at lags 6,12 , and 18 , probably generated by a nearby large period cycle, which make difficult to improve the forecast.

To summarize, we highlighted some situations in which the agents, through a simple observation of the time series of errors, can be able to improve their forecasts. However, in most situations, it can be very hard, and more refined forecasting mechanisms should be taken into account.

\section{CONCLUSIONS}

In this paper, we studied an OLG model with capital accumulation, logarithmic preferences, Cobb-Douglas technology, and agents working in both periods of life. We considered different types of expectations, focusing, in particular, on the effects on the dynamics of adaptive expectations. Our results indicate that, under both myopic and adaptive expectations, the qualitative properties of the dynamic behavior can be significantly different from that under perfect foresight. In fact, the dynamics may become rather complex with the emergence of cycles as the share of time devoted to labor when old increases, as well as chaotic dynamics can arise when it is large enough. Particularly significant is the role of adaptive expectations. In fact, we showed that even when the expectation mechanism allows recovering steady state stability, as with a suitably small expectation weight, the dynamics may not converge toward the steady state. In this case, there are multiple coexisting attractors introduced by the expectation mechanism itself. We may only have a "corridor stability."

A possible generalization of the present model consists in introducing an endogenous mechanism for $h$. It could be achieved by considering a similar approach to that proposed in Gori and Sodini (2011), obtaining a model in which the amount of labor supplied is exogenously set to 1 and endogenously determined when old. We notice that in Gori and Sodini (2011), it is assumed that, in both periods of life, the share of time to devote to labor and to pleasure is endogenously determined. In our case, since, in agreement with de la Croix and Michel (2002), we assume that agents always supply one unit of labor when young, the endogenization of $h$ would only concern the second period of life. Adopting a similar approach as in Gori and Sodini (2011) would lead to a sort of intermediate situation (in which the unit of labor supplied is exogenously equal to 1 when young and endogenously chosen when old) between the present model (in which in both periods of life the amount of labor is exogenously determined) and the model studied in Gori and Sodini (2011) (in which in both periods of life the amount of labor is endogenously determined). In Gori and Sodini (2011), the model is studied under perfect foresight. The study of such an "intermediate" situation between the present model and the model considered in Gori and Sodini (2011) would allow to better understand, under rational expectations, which is the role the amount of labor on the stability of the dynamics and on the occurrence of some phenomena as indeterminacy 
and multiple equilibria. Moreover, it would be interesting to also study the resulting model under myopic and/or adaptive expectations.

Another possible generalization concerns preferences and technology, considering a CIES-CES economy. The goal of the present paper was to show that complex dynamical behaviors are possible under nonrational expectations even in a simple Cobb-Douglas/logarithmic economy. It would be interesting to understand how such dynamics are affected by considering a more general economy. In this case, due to the analytical issues, the study should mostly rely on numerical investigations. Preliminary results show that the resulting dynamics are similar.

Finally, having noticed that, in general, it is difficult for the agents to improve their forecasts by observing the forecasting error time series, we aim to investigate the dynamics under more refined learning mechanisms, studying, for example, a least square learning mechanism similar to that in Hommes and Sorger (1998). We also aim to study the introduction of an evolutionary competition between the different heuristics, giving the possibility to the agents to switch between the different expectation mechanisms on the basis of some fitness measure.

\section{ACKNOWLEDGMENTS}

The authors wish to thank an anonymous reviewer for the valuable comments, which helped to improve the overall quality of the paper.

Bischi, G. I. and Naimzada, A., "Global analysis of a nonlinear model with learning," Econ. Notes 26, 143-174 (1997).

Bischi, G. I. and Naimzada, A., "A Kaleckian macromodel with memory," in Cycles, Growth, and the Great Recession (Routledge, 2014), pp. 103-116.

Blanchard, O. and Fisher, S., Lectures on Macroeconomics (The MIT Press, 1989).

Brooks, B. P., "Linear stability conditions for a first-order three-dimensional discrete dynamic," Appl. Math. Lett. 17, 463-466 (2004).
Bullard, J., "Learning equilibria,” J. Econ. Theory 64, 468-485 (1994).

Chen, H. and Li, M., "Productive public expenditure, expectation formations and nonlinear dynamics," Math. Soc. Sci. 56, 109-129 (2008).

Chen, H. and Li, M., "Child allowances, fertility, and chaotic dynamics," Chaos 23, 023106 (2013).

Chen, H., Li, M., and Lin, Y., "Chaotic dynamics in an overlapping generations model with myopic and adaptive expectations," J. Econ. Behav. Organ. 67, 48-56 (2008).

de la Croix, D. and Michel, P., "Myopic and perfect foresight in the OLG model," Econ. Lett. 67, 53-60 (2000).

de la Croix, D. and Michel, P., A Theory of Economic Growth: Dynamics and Policy in Overlapping Generations (Cambridge University Press, 2002).

Diamond, P., "National debt in a neoclassical growth model," Am. Econ. Rev. 55, 1126-1150 (1965).

Dohtani, A., Inaba, T., and Osaka, H., "Corridor stability of the neoclassical steady state," in Time and Space in Economics (Springer, 2007), pp. 129-143.

Duffy, J., "On learning and the nonuniqueness of equilibrium in an overlapping generations model with fiat money," J. Econ. Theory 64, 541-553 (1994).

Fanti, L. and Gori, L., "Public health spending, old-age productivity and economic growth: Chaotic cycles under perfect foresight," J. Econ. Behav. Organ. 78, 137-151 (2011).

Fanti, L. and Gori, L., "Fertility-related pensions and cyclical instability," J. Popul. Econ. 26, 1209-1232 (2013).

Gori, L. and Sodini, M., "Nonlinear dynamics in an OLG growth model with young and old age labour supply: The role of public health expenditure," Comput. Econ. 38, 261-275 (2011).

Gori, L. and Sodini, M., "Local and global bifurcations in an economic growth model with endogenous labour supply and multiplicative external habits," Chaos 24, 013122 (2014).

Grandmont, J., "On endogenous competitive business cycles," Econometrica 53, 995-1045 (1985).

Hommes, C., "Dynamics of the cobweb model with adaptive expectations and nonlinear supply and demand," J. Econ. Behav. Organ. 24, 315-335 (1994).

Hommes, C., "On the consistency of backward-looking expectations: The case of the cobweb," J. Econ. Behav. Organ. 33, 333-362 (1998).

Hommes, C. and Sorger, G., "Consistent expectations equilibria," Macroecon. Dyn. 2, 287-321 (1998).

Hommes, C. H., Behavioral Rationality and Heterogeneous Expectations in Complex Economic Systems (Cambridge University Press, 2013).

Leijonhufvud, A., "Effective demand failures," Swed. J. Econ. 75, 27-48 (1973).

Tuinstra, J., "Beliefs equilibria in an overlapping generations model," J. Econ. Behav. Organ. 50, 145-164 (2003). 